\title{
The Adsorbed Solute Transport with Diffusion Effects
}

\section{Khuzhayorov $\mathrm{B}^{1 *}$ and Mustofokulov $\mathrm{Z}^{2}$}

${ }^{1}$ Department of Mathematical Modelling, Samarkand State University, Samarkand, Uzbekistan

${ }^{2}$ Department of Radio electronica, Jizzakh Polytechnic Institute, Jizzakh, Uzbekistan

\begin{abstract}
In this paper the solute transport in a fractured-porous medium is considered with equilibrium adsorption. One the basis of numerical results an influence of adsorption on solute transport characteristics is estimated.
\end{abstract}

Keywords: Convective diffusion; Equilibrium adsorption; Fracturedporous media; Porous block; Relative mass transfer; Solute transport

\section{Introduction}

The problem of solute transport in fractured-porous media is encountered under various hydrogeological and ecological processes. A large number of papers have been devoted to the hydrodynamic modeling of the solute transport processes in a porous medium (PM) [1-6]. As a porous medium with a stagnant zone and certain degree of convention, can be considered as a fractured-porous medium (FPM) with a low permeability of porous blocks. In such FPM, liquid in porous blocks is considered to be stationary, and the system of fractures along which the liquid moves is regarded as a transit pore [7-9].

\section{Objects and Methods of Investigation}

In this paper, using the model approach [3-6], the motion of a dispersed liquid and the adsorbed solute transport into a FPM are examined taking into account the convective diffusion and dispersion effects. The main attention is paid to longitudinal convective diffusion in fracture and its effect on the adsorption of solute.

In order to investigate the solute transport in such media, we consider an element of a FPM consisting of a single fracture and an adjacent porous block (Figure 1). A fracture is a semi-infinite onedimensional object, so that the solute distribution and the fluid flow along its cross-section are considered as a homogeneous. In such case, the second fracture measurement, i.e. its thickness is not taken into account. The porous block occupies the first quarter of the plane Figure 1. Thus, the region $R\{0 \leq x<\infty, 0 \leq y<\infty\}$ is considered, only.

In a fracture there is a convective-diffusion solute transport and in a porous block only a diffusion one. Both in fracture and in porous

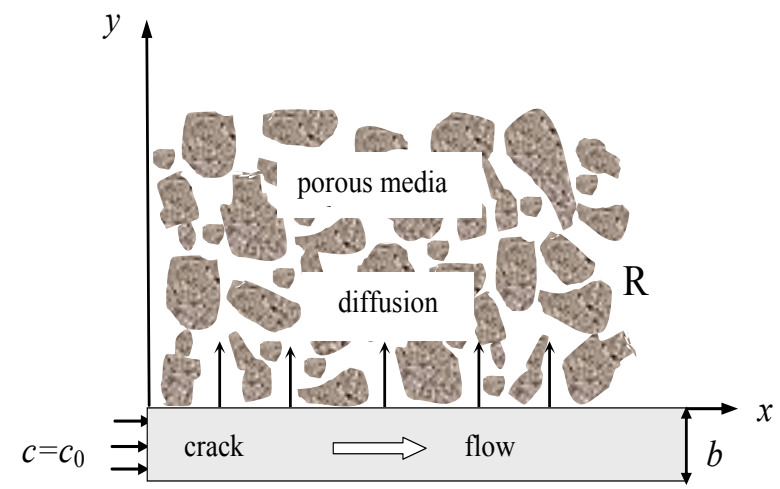

Figure 1: Schematic picture of the transfer of substance and flow in FPM. block, the solute can be adsorbed during the transport process.

The liquid in fracture flows at a given constant rate. At the end $(\mathrm{x}=0)$ of fracture, the liquid is supplemented by solute with concentration $\mathrm{c}_{0}$. Initially, the fracture and the porous block are considered to be filled with pure (without substance) liquid.

The fracture is modeled as a one-dimensional object, so the distribution of concentration along its cross-section is not considered. In fracture, the coefficient of convective diffusion $D_{f}^{*}$ must be considered as:

$$
D_{f}^{*}=D^{*}+\eta \frac{w}{\theta}
$$

where $D^{*}$ is the coefficient of molecular diffusion of the solute (liquid), $\eta$ the scattering coefficient, $\frac{w}{\theta}$ the physical velocity of the fluid. In fracture, the fluid velocity and the speed of filtration coincide, because $\theta=1$. Then, we have $D_{f}^{*}=D^{*}+\eta v$, where $v$ is the velocity of the fluid.

The balance equation of the solute transport process and the fluid flow in a FPM with convective diffusion and adsorption can be written as:

$$
\begin{aligned}
& b\left(\frac{\partial c_{f}}{\partial t}+\rho \frac{\partial s_{f}}{\partial t}+v \frac{\partial c_{f}}{\partial x}\right)=b D_{f}^{*} \frac{\partial^{2} c_{f}}{\partial x^{2}}+\left.\theta_{m} D_{m}^{*} \frac{\partial c_{m}}{\partial y}\right|_{y=0}, 0 \leq x<\infty, \\
& \frac{\partial c_{m}}{\partial t}+\frac{\rho}{\theta_{m}} \frac{\partial s_{m}}{\partial t}=D_{m}^{*} \frac{\partial^{2} c_{m}}{\partial y^{2}}, 0 \leq y<\infty
\end{aligned}
$$

where $c_{f}=c_{f}(t, x)$ is the solute concentration in fracture, $\mathrm{m}^{3} / \mathrm{m}^{3} ; c_{m}=c_{m}(t, x, y)$ the concentration in a matrix, $\mathrm{m}^{3} / \mathrm{m}^{3} ; s_{f}=\mathrm{s}_{f}(t, x)$ the concentration of the adsorbed solute in fracture, $\mathrm{m}^{3} / \mathrm{kg} ; s_{m}=s_{m}(t, x, y)$ the concentration of adsorbed matter in a matrix, $\mathrm{m}^{3} / \mathrm{kg} ; D_{m}^{*}$ - effective diffusion coefficient in a matrix, $\mathrm{m}^{2} / \mathrm{s} ; \rho$ is the density of saturated medium, $\mathrm{kg} / \mathrm{m}^{3} ; b$ the fracture width, $\mathrm{m} ; \theta_{m}$ the matrix porosity coefficient, $t$ - time, $\mathrm{s}$.

We solve the system in eqns.(1) and (2) with the equilibrium adsorption (Henry isotherm):

*Corresponding author: Khuzhayorov B, Professor, Department of Mathematica Modelling, Samarkand State University, Samarkand, Uzbekistan, Tel: 99866 2391140; E-mail: taylanov@yandex.com

Received December 31, 2018; Accepted February 14, 2019; Published February 21, 2019

Citation: Khuzhayorov B, Mustofokulov Z (2019) The Adsorbed Solute Transport with Diffusion Effects. J Appl Computat Math 8: 430. doi: 10.4172/21689679.1000430

Copyright: $\odot 2019$ Khuzhayorov B, et al. This is an open-access article distributed under the terms of the Creative Commons Attribution License, which permits unrestricted use, distribution, and reproduction in any medium, provided the original author and source are credited. 


$$
\begin{aligned}
& \frac{\partial s_{f}}{\partial t}=k_{f} \frac{\partial c_{f}}{\partial t}, 0 \leq x<\infty, \\
& \frac{\partial s_{m}}{\partial t}=k_{m} \frac{\partial c_{m}}{\partial t}, 0 \leq y<\infty,
\end{aligned}
$$

where $k_{m}, k_{f}$ are the adsorption coefficients in a matrix and fracture, $\mathrm{m}^{3} / \mathrm{kg}$.

We assume that there is no solute flow at the boundaries $x=\infty$ of fracture and matrix. The equilibrium of concentrations $c_{m}$ and $c$ is ensured at the boundary. Under these conditions, the initial and boundary conditions have the form:

$$
\begin{aligned}
& c_{f}(0, \mathrm{x})=c_{m}(0, x, y)=0, \\
& c_{f}(t, 0)=c_{0}, \\
& c_{f}(t, x)=c_{m}(t, x)=c_{m}(t, x, 0, \\
& \frac{\partial c_{f}(t, \infty)}{\partial x}=0, \\
& \frac{\partial c_{m}(t, x, \infty)}{\partial y}=0,
\end{aligned}
$$

\section{Numerical Solution of the Problem}

The problem in eqns. (1) and (9) is solved by the finite differences method in eqn. (9). To do this, in the region $R$ we construct a grid

$$
\begin{gathered}
\omega_{h_{1} h_{2} \tau}=\left\{\left(t_{k}, x_{i}, y_{j}\right), t_{k}=\tau k, x_{i}=i h_{1}, y_{j}=j h_{2},\right. \\
k=\overline{0, K}, i=0,1, \ldots, j=0,1, \ldots, \tau=T / K\},
\end{gathered}
$$

where $h_{1}$ is the grid spacing in the $x$ direction, $h_{2}$ the grid spacing in the $y$ direction, $\tau$ the grid spacing in time, $T$ the maximum time during which the process is investigated, $K$ the number of grid intervals in time. Thus, in eqn. (1) is approximated on the grid $\omega_{\tau} \cup \omega_{h_{1}}$, and in eqn. (2) on the grid $\omega_{\tau} \cup \omega_{h_{1} h_{2}}$. To approximate in eqn. (1), we use an implicit "anti-flow" scheme, and for in eqn. (2) we use a purely implicit scheme or a scheme with an advance. For Henry isotherms this approximations have the form

$$
\begin{aligned}
& R_{f} \frac{c f_{i}^{k+1}-c f_{i}^{k}}{\tau}+V \frac{c f_{i}^{k+1}-c f_{i-1}^{k+1}}{h_{1}}=D_{f}^{*} \frac{c f_{i+1}^{k+1}-2 c f_{i}^{k+1}+c f_{i-1}^{k+1}}{h_{1}^{2}}+\frac{\theta_{m}}{b} D_{m}^{*} \frac{c m_{i, 1}^{k}-c m_{i, 0}^{k}}{h_{2}} \\
& R_{m} \frac{c m_{i, j}^{k+1}-c m_{i, j}^{k}}{\tau}=D_{m}^{*} \frac{c m_{i, j-1}^{k+1}-2 c m_{i, j}^{k+1}+c m_{i, j+1}^{k+1}}{h_{1}^{2}}, i=\overline{1, I}, j=\overline{1, J}, k=\overline{0, K-1},
\end{aligned}
$$

where, $\quad R_{f}=1+\rho k_{f}, R_{m}=1+\frac{\rho}{\theta_{m}} k_{m}, c f_{i}^{k}, c m_{i, j}^{k}$ are grid values of concentrations $c_{f}(t, x)$ and $c_{m}(t, x, y)$ at grid points $\left(t_{k}, x_{i}\right)$ and $\left(t_{k}, x_{i} y_{j}\right)$, respectively. In eqn. $(10,11)$ are reduced to three-point grid equations

$$
\begin{aligned}
& A_{1} c f_{i-1}^{k+1}-B_{1} c f_{i}^{k+1}+C_{1} c f_{i+1}^{k+1}=-F_{i}^{(1)}, i=\overline{1, I-1}, k=\overline{0, K-1} \\
& A_{2} c m_{i, j-1}^{k+1}-B_{2} c m_{i, j}^{k+1}+C_{2} c m_{i, j+1}^{k+1}=-F_{i, j}^{(2)}, i=\overline{0, I}, j=\overline{1, J-1}, k=\overline{0, K-1}
\end{aligned}
$$

where $I, J$ sufficiently large integers, taken in such a way that the concentration and adsorption fronts do not reach the points, $x_{I},\left(x_{I}, y_{J}\right), \quad A_{1}=\frac{D_{f}^{*}}{h_{1}^{2}}+\frac{V}{h_{1}}, \quad C_{1}=\frac{D_{f}^{*}}{h_{1}^{2}}, \quad F_{i}^{(1)}=\frac{R_{f}}{\tau} c f_{i}^{k}+\frac{\theta_{m} D_{m}^{*}}{b h_{2}}\left(c m_{i, 1}^{k}-c m_{i, 0}^{k}\right)$, $A_{2}=\frac{D_{m}^{*}}{h_{2}^{2}}, B_{2}=2 \frac{D_{m}^{*}}{h_{2}^{2}}+\frac{R_{m}}{\tau}, C_{2}=\frac{D_{m}^{*}}{h_{2}^{2}}, F_{i, j}^{(2)}=\frac{R_{m}}{\tau} c m_{i j}^{k}$.

The initial and boundary conditions are approximated in the form

$$
c f_{i}^{0}=c m_{i, j}^{0}=0 \text {, }
$$

$$
\begin{aligned}
& c f_{0}^{k+1}=c_{0}, \\
& c f_{i}^{k+1}=c m_{i, 0}^{k+1}, \\
& c f_{I}^{k+1}=c f_{I-1}^{k+1}, \\
& c m_{i, J}^{k+1}=c m_{i, J-1}^{k+1},
\end{aligned}
$$

To solve the difference in eqn. $(12,13)$, we apply the sweep method. So, the solution can be presented in the form

$$
\begin{aligned}
& c f_{i}^{k+1}=\alpha_{i+1}^{(1)}+\beta_{i+1}^{(1)} c f_{i+1}^{k+1}, \quad i=\overline{1, I-1}, k=\overline{0, K-1}, \\
& c m_{i j}^{k+1}=\alpha_{j+1}^{(2)}+\beta_{j+1}^{(2)} c m_{i, j+1}^{k+1}, \quad i=\overline{0, I-1}, j=\overline{0, J-1}, k=\overline{0, K-1},
\end{aligned}
$$

where $\alpha_{i+1}^{(1)}, \beta_{i+1}^{(1)}, \alpha_{j+1}^{(2)}, \beta_{j+1}^{(2)}$ are the run ratios. Using in eqn. (19) from (12), we obtain the following recurrence formulas to determine the running coefficients $\alpha_{i+1}^{(1)}, \beta_{i+1}^{(1)}$ :

$$
\alpha_{i+1}^{(1)}=\frac{F_{i}^{(1)}+A_{1} \cdot \alpha_{i}^{(1)}}{B_{1}-A_{1} \cdot \beta_{i}^{(1)}}, \quad \beta_{i+1}^{(1)}=\frac{C_{1}}{B_{1}-A_{1} \cdot \beta_{i}^{(1)}}, \quad i=\overline{0, I-1} .
$$

In a similar way, we obtain recurrence formulas for the running coefficients in (20)

$$
\alpha_{j+1}^{(2)}=\frac{F_{i, j}^{(2)}+A_{2} \cdot \alpha_{j}^{(2)}}{B_{2}-A_{2} \cdot \beta_{j}^{(2)}}, \quad \beta_{j+1}^{(2)}=\frac{\tilde{N}_{2}}{B_{2}-A_{2} \cdot \beta_{j}^{(2)}}, \quad j=\overline{0, J-1} .
$$

To determine the starting values of the coefficients, we use the boundary conditions in eqn. $(15,16)$. From in eqn. (19) we have $c f_{0}^{k+1}=\alpha_{1}^{(1)}+\beta_{1}^{(1)} c f_{1}^{k+1}=c_{0}$, whence $\alpha_{1}^{(1)}=c_{0}, \quad \beta_{1}^{(1)}=0$.

From in eqn. (20) for $j=0$, We obtain $\mathrm{cm}_{i, 0}^{k+1}=\alpha_{1}^{(2)}+\beta_{1}^{(2)} \mathrm{Cm}_{i, 1}^{k+1}=c f_{i}^{k+1}$, so $\alpha_{1}^{(2)}=c f_{i}^{k+1}, \quad \beta_{1}^{(2)}=0$.

The calculation is carried out in the following sequence: In eqn. (19) we determine $c f_{i}^{k+1}$ by the sweep method, then in eqn. (20) $\mathrm{cm}_{i, j}^{k+1}$ by same method.

After determining the concentration fields from the isotherms, it is possible to determine the adsorption fields

$$
s f_{i}^{k+1}=k_{f} c f_{i}^{k+1}, \quad s m_{i, j}^{k+1}=k_{m} c m_{i, j}^{k+1} .
$$

For the above procedure to calculate the fields $c m_{i, j}^{k}, c f_{i}^{k}$ and $s m_{i, j}^{k}, s f_{i}^{k}$, as well as the relative mass transfer from the fractures $Q=-\left.\theta_{m} D_{m}^{*} \frac{\partial c_{m}}{\partial y}\right|_{y=0}$, a special computational program is compiled into the matrix.

In practical calculations, the following values of the initial parameters were used: $c_{0}=0,01, \theta_{m}=0,2, D_{m}{ }^{*}=1 \cdot 10^{-6} \mathrm{~m}^{2} / \mathrm{s}, \mathrm{b}=4.10^{-4}$ $\mathrm{m}, k_{m}=3.10^{-5} \mathrm{~m}^{3} / \mathrm{kg}, k_{f}=3.10^{-5} \mathrm{~m}^{3} / \mathrm{kg}, \rho=2500 \mathrm{~kg} / \mathrm{m}^{3}$.

\section{Results and its Discussion}

Figure 2 reflects the surface of relative concentration $\mathrm{z}$ and adsorption $s$. One can see that on surfaces, an increase in solute concentration in fracture and, accordingly, in the region $R$, with a small $x$ the solute transport from the fractures into the porous blocks becomes significant. The longitudinal convective diffusion in fracture leads to a smearing of the profiles $c_{\rho}$, which in turn affects the distribution of $c_{m}$ Figure 2a. In turn, convective diffusion leads to an increase in adsorption in the fracture and the porous block Figure $2 b$, as well as 
Citation: Khuzhayorov B, Mustofokulov Z (2019) The Adsorbed Solute Transport with Diffusion Effects. J Appl Computat Math 8: 430. doi: 10.4172/2168-9679.1000430

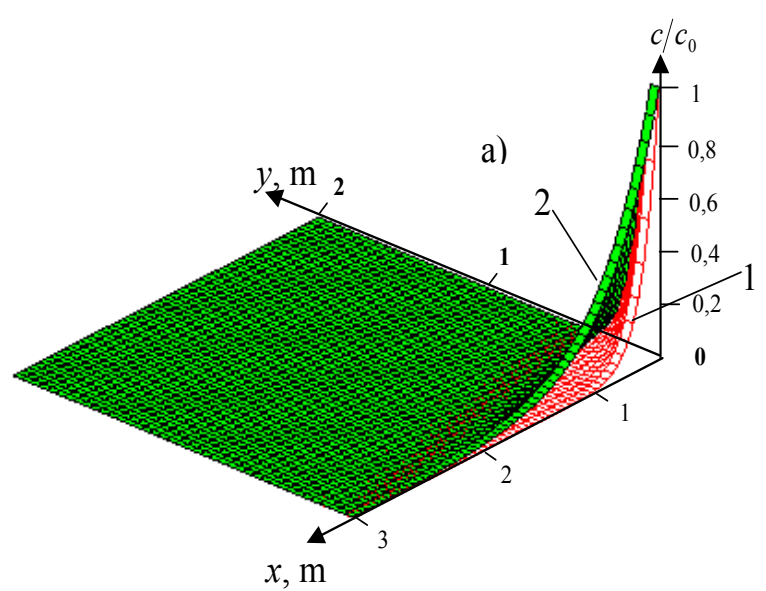

Figure 2a: The surface of the relative concentration $c / c_{0}(a)$.

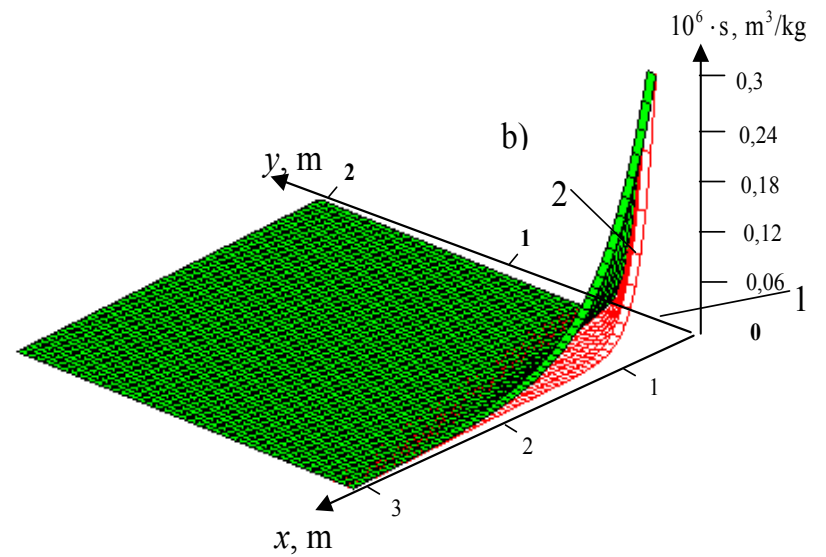

Figure 2b: The surface of the relative concentration at $v=5 \cdot 10^{-4} \mathrm{~m} / \mathrm{s}, t=10000$ S, $D_{f}^{*}=0(1), D_{f}^{*}=6 \cdot 10^{-4} \mathrm{~m}^{2} / \mathrm{s}(2)$.

mass exchange between the fracture and the porous block.

The results of calculations of the relative mass transfer $Q$ from a fracture to a porous block are shown in Figure 3a. Analysis of the graphs shows that for a certain value of time the increase in the value of convective diffusion in fracture leads to an increase in $Q$ through $y=0$. Based on the curves in Figure $3 \mathrm{a}$ was also estimated cumulative conditional mass transfer $Q$ through $y=0$.

$$
Q_{\text {cum }}=\int_{0}^{\infty} Q d x
$$

For each time point $t$ that characterizes the total mass transfer through $y=0$ at a given time. In Figure $3 \mathrm{~b}$ shows graphs of the change $Q_{\text {cum }}$ in time $t$. It can be seen that the cumulative conditional mass transfer first increases in time, then a monotonic decrease its value is observed. At the same time, with an increase in the value of convective diffusion in the fracture $Q_{\text {cum }}$, the growth rate is more accelerated.

In Figure $3 c$, shows the graphs of the total conditional solute transport change through $y=0$ from fracture to porous block versus time.

$$
Q_{\text {sum }}=\int_{0}^{t} Q_{\text {cum }} d t=\int_{0}^{t} \int_{0}^{\infty} Q d x d t
$$
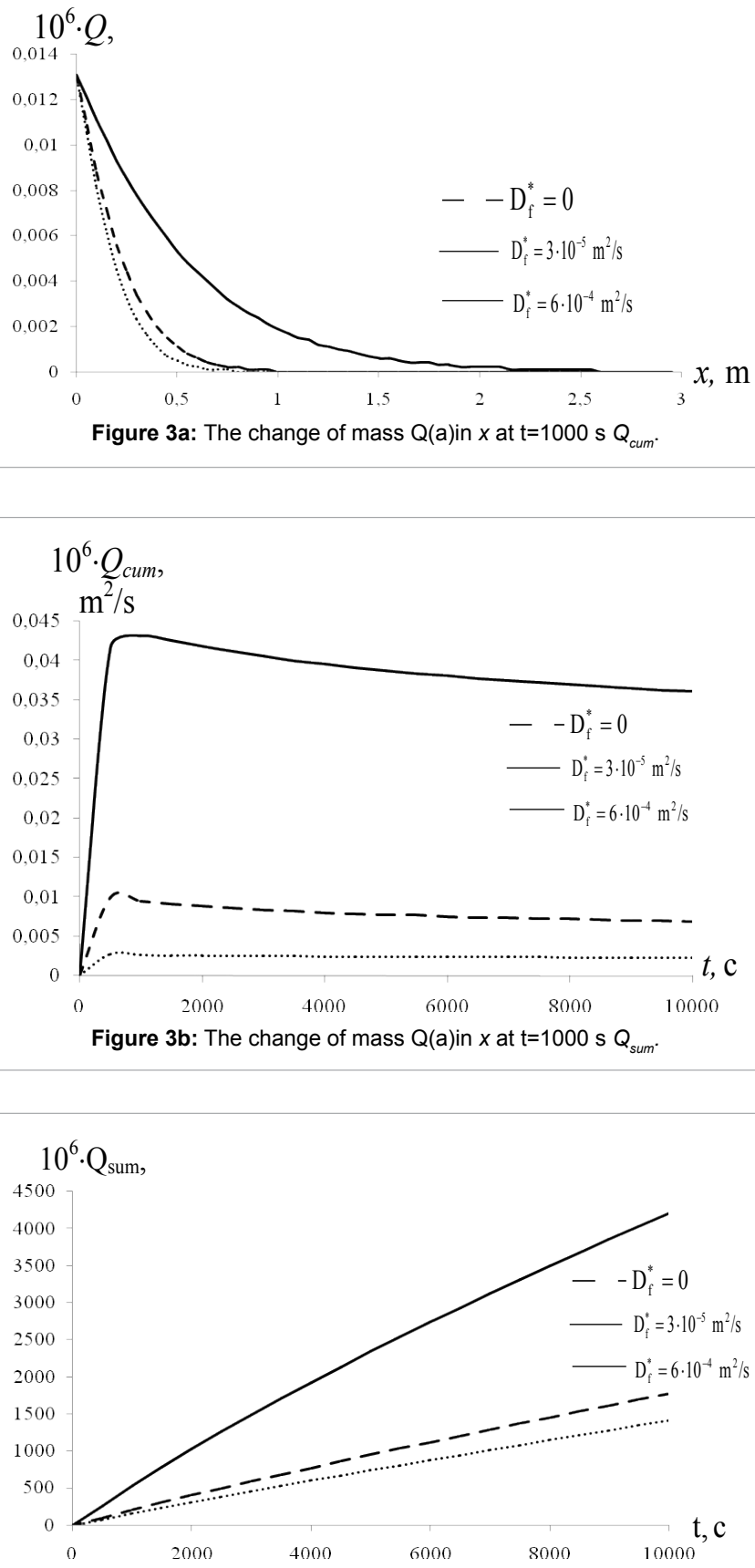

Figure 3c: The change of mass $Q(a)$ in $x$ at $t=1000 \mathrm{~s}$ on $\mathrm{t}$ at $v=5.10^{-4} \mathrm{~m} / \mathrm{s}$, for various values of $D_{f}^{*}$.

The graphs show that $Q_{\text {cum }}$ increases monotonically with time. In this case, an increase in the value of convective diffusion in the fracture contributes to an increase $Q_{\text {cum }}$

The results of calculations for different values of the velocity of motion a $\eta=2.10^{-3} \mathrm{~m}, \mathrm{D}^{*}=1.10^{-6} \mathrm{~m}^{2} / \mathrm{s}$ in fracture are shown in Figures 4 and 5. In Figure 4, the surfaces $c / c_{0}$ are also reflected $s$ for two values $v$. On surfaces it can be seen that an increase in the velocity of the fluid in a fracture leads to an increase in the distribution of concentration in the region $R$, which in turn increases the adsorption of the substance.

The nature of the change in the relative mass transfer $Q$ is shown 
Citation: Khuzhayorov B, Mustofokulov Z (2019) The Adsorbed Solute Transport with Diffusion Effects. J Appl Computat Math 8: 430. doi: 10.4172/2168-9679.1000430

Page 4 of 4

in Figure 5a. For $v=2.10^{-3} \mathrm{~m} / \mathrm{s}$, the relative mass transfer $Q$ is higher in the whole region $x$ than in $v=1.10^{-3} \mathrm{~m} / \mathrm{s}$ and $v=5.10^{-4} \mathrm{~m} / \mathrm{s}$. This pattern

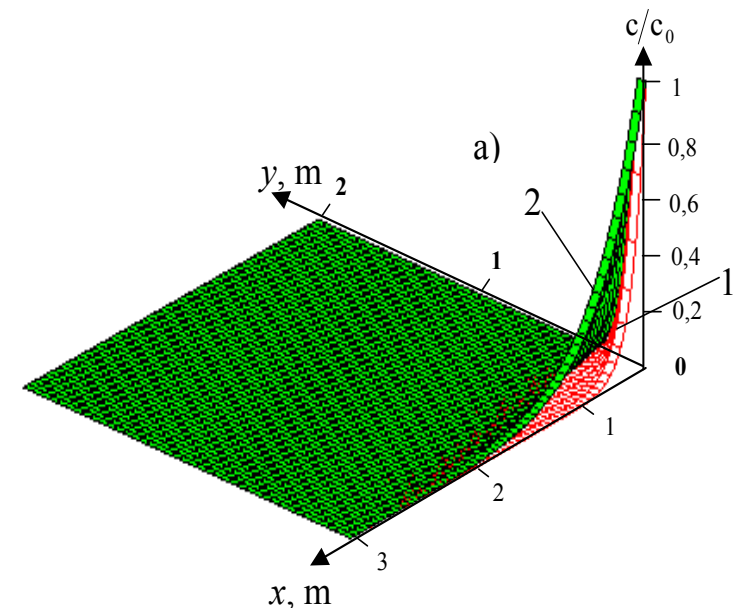

Figure 4a: The surface of the relative concentration $c / c_{0}$ and adsorbed concentrations.

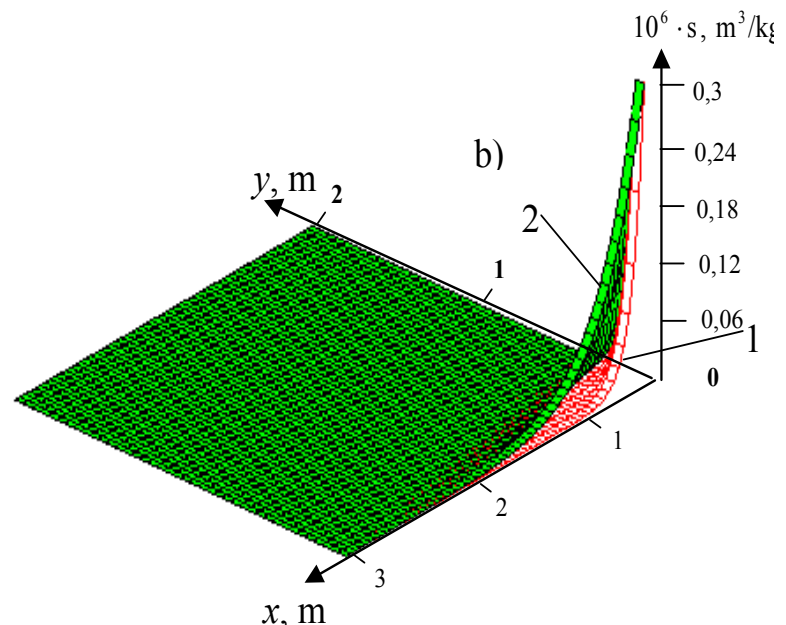

Figure 4b: The surface of the relative concentration $c / c_{0}$ at $t=5000 \mathrm{~s}, v=5.10^{-4}$.

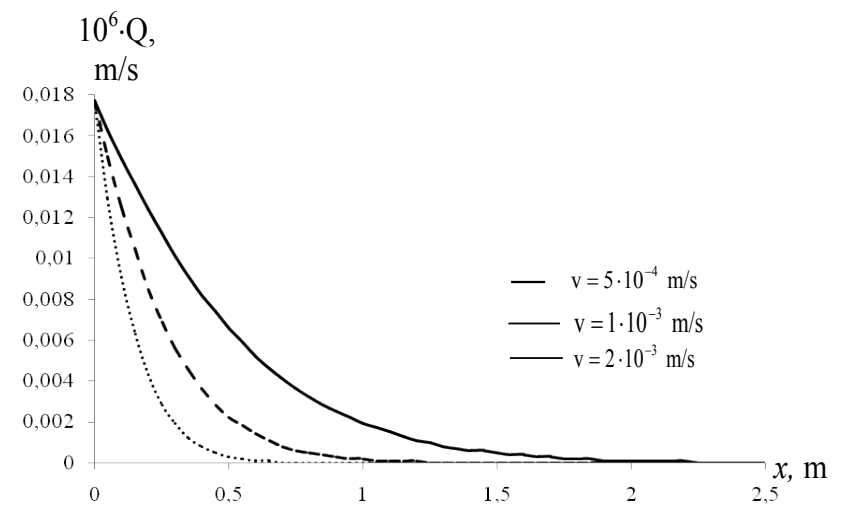

Figure 5: The different values of the velocity of motion a $\eta=2.10^{-3} \mathrm{~m}, D^{*}=1.10^{-6} \mathrm{~m}^{2} / \mathrm{s}$. is observed for the cumulative and relative flow from the fracture to the porous block (Figure 5). At the same time, with increasing speed, movement in a fracture leads to an increase in $Q_{c u m}$ and $Q_{c u m}$.

\section{Conclusion}

We conclude that an increase of the convective diffusion coefficient leads to an acceleration of the process of the formation of an equilibrium adsorption regime as well as the distribution of concentration in a porous medium. Comparing the data presented, it can be seen that the diffusion rate leads to an increase in the concentration distribution zone, however, higher concentration gradients are formed at the boundary of the fracture and porous block.

\section{References}

1. Nikolaevsky VN, Bondarev EA, Mirkin MI, Stepanova GS, Terzi VP(1968) Movement of hydrocarbon mixtures in a porous medium, p: 192.

2. Fetter CW(1999) Contaminant Hydrology. Prentice-Hall, Upper Saddle River NJ, USA.

3. Grisak GE, Pickens JF, Cherry JA (1980) Solute transport through fractured media: 1. The effect of matrix diffusion. Water Resources Research 16: 719730 .

4. Genuchten MTV, Davidson JM, Wierenga PJ (1974) An Evaluation of Kinetic and Equilibrium for the Prediction of Pesticide Movement Porous Media. Soil Science Society of America Journal 40: 29-35.

5. Genuchten MTV, Wagenet RJ (1989) Two-Site/Two-Region Models for Pestiside Transport and degradation: Theoretical Development and Analytical Solutions. Soil Science Society of America Journal 53:1303-1310.

6. Grisak GE, Pickens JF (1981) An analytical solution for solute transport through fractured media with matrix diffusion. Journal of Hydrology 52: 47-57.

7. Habbar A, Kolditz O (1998) Numerical modelling of reactive transport processes in fractured porous media. Proceedings of the GQ'98 Conference held at Tubingen, Germany, September 1998, IAHS Publ.No: 250.

8. Neville CJ (2006) Solute Transport in Fractured-Porous Media: Dual Porosity Simulations with MT3D. Papadopulos \& Associates, Inc.

9. Samarskii AA (2001) The Theory of difference schemes. Taylor \& Francis Inc New York, United States, p: 786. 